\title{
Una reflexión sobre el paradigma de la integralidad en el transporte público de Bogotá ${ }^{1}$
}

A reflection on the paradigm of integrality in the public transport of Bogotá

Juan Manuel González Trujillo

Recepción: 8 de agosto de 2016 Aprobación: 20 de enero de 2017

\section{Resumen}

El presente artículo expone los resultados de la investigación que tuvo por finalidad analizar el paradigma de la integralidad en el transporte público en Bogotá, a partir de un enfoque cualitativo-descriptivo basado tanto en estudios de revisión documental sobre el tema, como en entrevistas semiestructuradas a fuentes institucionales. La finalidad de la reflexión que se expone aquí es comprender la desarticulación en la planificación y gestión del transporte público masivo, en distintos niveles, respecto del paradigma de la integralidad, y sustentar la necesidad de intensificar esfuerzos para fortalecerlo.

Palabras clave: transporte público, paradigma de integralidad, política pública, sistema integrado de transporte público.

1 Artículo producto del proyecto de investigación: 'El paradigma de la integralidad en el transporte público de Bogotá" enmarcado dentro de la modalidad de Gestión de grandes proyectos urbanos en espacios metropolizados: sistemas de transporte masivo de la Universidad Piloto de Colombia sede Bogotá.

2 Arquitecto y Magíster en Gestión Urbana de la Universidad Nacional. Docente investigador de la Facultad de Ingeniería Civil de la Universidad Santo Tomás, sede Villavicencio. Correo electrónico: juanmgonzalez@ usantotomas.edu.co 


\begin{abstract}
This article presents the results of an investigation that was aimed at analyzing the paradigm of integrity in public transport in Bogota, from a qualitative descriptive approach was based both on literature review of studies on the subject, as in interviews semi-structured institutional sources. The purpose of reflection as set forth in this text is to understand the disruption in the field of planning and management of mass transit, at different levels, based on the paradigm of completeness, and support the need to intensify efforts to strengthen it.
\end{abstract}

Key words: public transport paradigm of integrity, public policy, integrated public transport system.

\title{
INTRODUCCIÓN
}

El transporte público es un factor determinante de la movilidad. Esta afirmación

ofrece una perspectiva de los individuos en su realidad socioeconómica y espacial (edad, género, categoría sociolaboral) más amplia que el término transporte, el cual se limita a una relación de oferta y demanda expresada esquemáticamente, por un lado, en cantidad de infraestructuras y medios de transporte y, por el otro, en el número de desplazamientos por día, modo, itinerario, tiempo (Montezuma, 2003, pág. 177).

El transporte público y el tráfico están asociados a los factores económicos y técnicos que condicionan los desplazamientos de las personas, en tanto que la movilidad se centra en los individuos y su entorno. Aunque son dimensiones diferentes, es claro que una adecuada planificación del transporte tiene una positiva incidencia en la movilidad. Esta nueva concepción del transporte público se materializa en las recientes políticas, planes y proyectos en Bogotá — por lo menos en el discurso- bajo el concepto de integralidad o integración: un transporte integral o integrado en el que se articulan diversos medios de transporte, acorde con el ordenamiento territorial en el ámbito de la ciudad-región. Así pues, se ha intentado superar la concepción del transporte público como simple satisfactor de las condiciones de la oferta y la deman- 
da de desplazamientos, basadas en la delimitación de la población económicamente activa, para reconocer su importancia en la transformación de la estructura socioeconómica y del espacio social con base en la participación de la población.

En concordancia con lo dispuesto en el párrafo anterior, y en el marco de la responsabilidad en el servicio de transporte, es importante anotar que el problema de la responsabilidad civil no es un problema de conciencia, sino un problema de orden económico, no se trata de castigar, sino de reparar y de indemnizar el daño causado (Woolcott, 2016, p.33)

Este artículo presenta los resultados de la exploración del concepto de integralidad e integración en los documentos de planificación del transporte público y del desarrollo urbano, así como en los testimonios de gestores institucionales relacionados con este ámbito, para establecer relaciones entre este y los tres tipos de integración que se presentan actualmente en las perspectivas de desarrollo de la ciudad: 1) integración regional (en la que el transporte se convierte en un articulador entre municipios en proceso de metropolización), 2) integración intersectorial (entendida como la planificación articulada del transporte con otros sectores como la planificación territorial) y 3) integración multimodal (entendida como la articulación física y/o virtual entre modos de transporte público), y examina, a nivel conceptual, la relación entre la gestión urbana y la planificación del transporte público en una escala supramunicipal, teniendo en cuenta los procesos de metropolización, por lo cual concibe los sistemas integrados de transporte masivo (SITM) como grandes proyectos urbanos cuyo esquema de gestión es más complejo que el de una simple obra de infraestructura y requiere de una integración entre agentes de muy distintos tipos, desde distintos niveles de gobierno - nación, municipios - hasta el sector público y privado, pasando por la integración entre autoridades locales de varios sectores (planificación, transporte, hacienda).

Se parte, para su desarrollo, de la idea de que es relevante ahondar la comprensión del concepto de integralidad en la planificación del transporte público y analizar las dimensionalidades que dan lugar a diversos tipos de integración para el desarrollo de la ciudad y la región, a fin de verificar si este concepto está presente en las prácticas de planificación y gestión de este tipo de proyectos de sistemas de transporte público en la ciudad y, en caso de que no lo esté, sustentar la pertinencia de su adopción, con 
base en los resultados del seguimiento que se hizo en el trabajo investigativo de los proyectos de transporte público y movilidad, desde los conceptos abstractos hasta los planes y proyectos concretos. También se busca visibilizar los avances y limitaciones que presenta la ciudad en el camino hacia la consolidación de un transporte urbanoregional que funcione realmente como sistema integrado.

\section{FaCtores de la PLANificación y GESTIÓN DEL TRANSPORTE PÚBLICO}

A pesar de que como plantean expertos en la economía del transporte, entre ellos Campos, De Rus \& Numbela (2003), los elementos y principios económicos que rigen las modalidades de este, y del transporte público específicamente, no han sufrido cambios relevantes en su funcionamiento en la época actual, la industria y el sector de sus servicios sí han atravesado por importantes transformaciones, producto en gran medida de los procesos de metropolización, de la globalización y de la descentralización política, que se han registrado en la región de América Latina y el Caribe en las últimos décadas, lo que no solo ha significado, en este y otros sectores, nuevos retos en la gobernabilidad y la relación entre los diversos actores públicos y privados, sino también una reflexión sobre la lógica de los mecanismos clásicos de la planificación urbana (caracterizados por centrarse en el control de uso de la ciudad y el territorio) y sobre su capacidad de dar respuesta a estas transformaciones y sus nuevas demandas.

A esta situación se suman los procesos de tercerización de la economía y de privatización que ha afectado a la gestión pública, lo que conlleva a cambios importantes en la institucionalidad, las modalidades de gestión y las políticas urbanas. En Colombia, estos cambios se han sentido con mayor rigor en los municipios, producto de la descentralización política, pues han debido asumir responsabilidades en la política urbana para enfrentar la competitividad, en la mayoría de los casos con déficit en sus capacidades instrumentales, administrativas y técnicas.

En este panorama, juega un papel protagónico el transporte público en tanto que un instrumento de la transformación urbana que, según Montezuma, se ha encontrado estrechamente vinculado en América Latina con la urbanización (2003, p. 185), esto es, con los usos del suelo y el ordenamiento de la ciudad. Tanto así que los sistemas 
de transporte público han determinado la evolución de diversas tipologías de ciudad, por ejemplo, de la ciudad lineal orientada por el tranvía, a la ciudad radial en la que domina el autobús, lo que no quiere decir que esta vinculación se haya dado de forma organizada; por el contrario, según este autor, los centros urbanos de la región se caracterizan por haberse expandido con una deficiente o inexistente planificación urbana y por una falta de control sobre el suelo que ha permitido que muchos de los asentamiento urbanos de los países de la región alcancen grandes dimensiones, bien por su extensión en el territorio o bien por su densidad demográfica (Montezuma, 2003, p. 177).

En relación con esto, las infraestructuras de transporte público y los servicios se han adaptado a las demandas de ciudad, dentro de los límites que les han impuesto las posibilidades tecnológicas y la capacidad financiera, entre otros factores, como señala Silva, para quien el ciclo de uso del suelo y el sistema de transporte, en el caso de la expansión urbana en Bogotá, determinó la consolidación del centro como el foco más importante de esta, expandiéndose a un territorio mayor y desplazando actividades como la vivienda a lugares periféricos(2010, p. 41), en los cuales la accesibilidad ofrecida por el transporte público tradicional se hizo más limitada, debido a la poca demanda que se polarizaba, naturalmente, en las centralidades, proceso al que se sumó el aumento de la densidad, del valor del suelo y de la renta en estas. La accesibilidad a la periferia estaba sujeta a las condiciones que ofrecía un sistema de transporte público desorganizado y dominado por un régimen de competencia en los principales corredores arteriales de la ciudad que concentraban la mayor parte de demanda, en un círculo vicioso (2010).

De acuerdo con Silva, (2010) esta fue la situación que se propuso enfrentar Plan de Ordenamiento Territorial (POT), acogiendo el Régimen de Ordenamiento Territorial (Ley 388 de 1997), al reconocer que gran parte de los problemas de congestión y acceso al transporte en la periferia se debía a la lógica de consolidación del centro o centros que se había dado en la ciudad (2010, pp. 42-43), para lo cual se determinó como principal función del sistema de transporte público la de integrar la red de centralidades con los distintos polos de actividad.

Para Silva, con esta normativa se hace evidente la relación entre los usos del suelo y el transporte público en Bogotá, pues en esta el transporte adquiere una función 
importante en la planificación del desarrollo de la ciudad, que posteriormente fue ratificada por el Plan Maestro de Movilidad (PMM) en el artículo 19: "Integrar de manera jerarquizada e interdependiente los diferentes modos de transporte con las infraestructuras de movilidad, teniendo como eje estructurador el sistema de transporte público" (Alcaldía Mayor de Bogotá, 2006).

Los anteriores planteamientos permiten justificar la relación entre la gestión urbana y la planificación del transporte público en Bogotá y, si a esto se le suma que tanto el Plan de Ordenamiento Territorial como el Plan Maestro de Movilidad establecen que el sistema de transporte público tiene como ejes estructuradores los diferentes modos de transporte masivo, metro, tranvía, tren de cercanías y buses por carriles exclusivos, entendidos como un conjunto de modos que se configuran en la propuesta de un sistema integrado de transporte público (Silva, 2010, p. 46), se sustenta la pertinencia de interrogarse por la integralidad desde la dimensión de un paradigma que señale los atributos deseables en un sistema de transporte público y en sus relaciones con otras dimensiones, toda vez que proponer un sistema integrado de este tipo puede revelar la idea de ciudad a la que le están apuntando las iniciativas de desarrollo de los distintos actores y gestores de la sociedad.

En el marco de lo dispuesto en líneas anteriores, es importante hacer referencia a los profesores Rodríguez y Carvajal, en lo referente al contexto de lo que supone la noción de urbanización. Al respecto los citados doctrinantes han considerado a la urbanización como "una forma tecnológica específica, expresión técnica de las relaciones de producción capitalista, gracias a la cual se logra reinvertir la relación existente con la tierra y el territorio, transformando su función de laboratorio de la producción, para mero soporte de una actividad que se reorganiza fuera de ella" (Rodríguez \& Carvajal, 2015, p. 156).

\section{El PARADIGMA DE LA INTEGRALIDAD EN LA GESTIÓN DEL TRANSPORTE PÚBLICO Y LAS DIMENSIONES DE LA INTEGRACIÓN}

En un sentido general, la palabra integralidad proviene del verbo del latín integer que significa entero. En el sentido de prescripción que caracteriza al término "integralidad", acoge lo que debe estar unido o completo, dado que las partes que lo configu- 
ran están solidariamente organizadas, esto es, son diferentes, pero dependen las unas de las otras, son complementarias (Muñoz \& Alvarado, 2009, p. 108),

el concepto de integralidad parte necesariamente de la idea de una realidad que se percibe como unidad y complejidad. Es decir, se entiende que la realidad se caracteriza en su esencia como un sistema conformado por partes o elementos que se asocian e interactúan para el logro de ciertos fines. De ahí que cada elemento no pueda explicarse sino en función de los otros (Muñoz \& Alvarado, 2009,109).

Tal concepto, aplicado al transporte, implica el reconocimiento de que este, definido como "el movimiento de personas y mercancías a lo largo del espacio mediante tres modos principales: terrestre, aéreo o marítimo, o alguna combinación de estos" (Campos, De Rus \& Nombela, 2003, p. 3), se basa en la relación entre dos actividades: la construcción y explotación de infraestructura, y los servicios de transporte que, aunque diferentes, son interdependientes, pues según, Campos, Rus \& Nombela, la industria de transporte está conformada por las empresas que se dedican a estas actividades y, por lo tanto, podría pensarse que, puesto que producen el mismo tipo de servicio, deberían constituir un conjunto más o menos homogéneo (2003, p. 3), aunque las diferencias entre los modos de transporte hagan que las industrias particulares suelan especializarse en sus ámbitos específicos, sin interesarse o reconocer el papel de las demás, respecto de lo cual estos autores afirman que "a pesar de las diferencias y separación entre modalidades de transporte, existen unas características comunes que desde la perspectiva del análisis económico las hace susceptibles de un tratamiento conjunto y sistemático" (2003, p. 2).

Sin embargo, no solo el análisis de la industria del transporte y sus servicios señala la integralidad como un principio fundamental. Desde hace más de tres décadas la Comisión Económica para América Latina y el Caribe (Cepal), a través de la Unidad de Servicios de Infraestructura (que originalmente se denominó División de Transporte de la Cepal) ha venido haciendo importantes reflexiones sobre el papel del transporte en el desarrollo de la región, que ha servido de base en la creación de políticas y programas sobre el tema, por ejemplo, los que se han derivado de los estudios para sustentar un marco institucional, operativo y normativo del transporte internacional de carga y pasajeros en la región; reflexiones que han terminado por proponer la in- 
tegralidad como una paradigma en la planificación del transporte. Según Sánchez, el trabajo de la Cepal sobre este tema ha sustentado que:

Es fundamental que los gobiernos de América Latina y el Caribe mejoren y fortalezcan su institucionalidad, incrementado la coordinación y coherencia al interior del propio Estado y fortaleciendo la relación con el sector privado a través de marcos modernos que contengan un equilibrio entre el planeamiento, evaluación, capacidad y maduración de las inversiones, y cuyo principal foco sea el desarrollo integral de la economía donde se inserten los servicios de infraestructura y transporte, por sobre los aspectos financieros involucrados (Sánchez, 2010, p. 3).

En esta anterior postura, Sánchez recoge la propuesta presentada en 2008, para la Cepal, por Gabriel Pérez, en la que este autor sustenta que la mejora en las condiciones de desarrollo económico y social de la región depende, en gran medida, de incorporar en los emprendimientos en infraestructura de transporte un planteamiento integral que equilibre la participación de los diversos componentes y de sus roles. En este documento Pérez afirma que

tradicionalmente, las políticas públicas relativas a la infraestructura y el transporte se han visto de forma disociada, lo cual impide hacer provisión eficiente de bienes públicos, comunes y escasos. El transporte y las políticas asociadas son, muchas veces, analizadas e implementadas de forma unimodal. La logística, por su parte, es frecuentemente dejada de lado de cualquier planteamiento del transporte y de la infraestructura $(2008$, p. 2).

Según este autor, y a juicio de la Cepal, es necesario modificar este foco unimodal y adoptar políticas integrales que relacionen el desarrollo de la infraestructura, los servicios de transporte, la logística, la facilitación del comercio y el transporte sobre la base de un desarrollo urbano y social (Pérez, 2008, p. 3), y en dicho cambio de enfoque deben converger los diversos ámbitos y sectores, a través del diálogo y considerando, además de los aspectos económicos, los sociales y medioambientales.

Si vemos la integralidad como un paradigma y condición necesaria para todo sistema de trasporte, entonces se puede definir la integralidad "como la existencia de una 
planificación y ejecución coordinada entre los agentes públicos y de la sociedad civil, con una visión integradora para todos los modos de transporte que intervienen en la movilidad de los pasajeros, en la carga interna y la del comercio exterior, así como también de la logística que los articula"(Cipoletta, 2011, p. 2).

Esto implica infraestructura, logística, movilidad, control de mercancías y entidades de sector privado y público. Las estrategias para lograr este paradigma, dependen del reconocimiento de tres tipos de integración: 1) la integración regional, 2) la integración intersectorial y 3) la integración multimodal.

La integración regional se basa en la conformación de áreas metropolitanas, que en América Latina han estado determinadas por procesos de metástasis urbana, es decir, procesos de urbanización que no son producto de una adecuada planificación, sino de procesos sociales relacionados con la demanda de vivienda A partir de esta metástasis urbana se configuran ciudades metropolizadas sin planificación pero con grandes centros productivos. Estos procesos también han generado el surgimiento de las áreas metropolitanas que se entienden como entidades administrativas que se forman a partir de varios municipios reunidos alrededor de un municipio y relacionada social y económicamente.

De acuerdo con el artículo 1 de la Ley Orgánica de Áreas Metropolitanas (Ley 128 de 1994) se definen como "formadas por un conjunto de dos o más municipios integrados alrededor de un municipio núcleo o metrópoli, vinculados entre sí por estrechas relaciones de orden físico, económico y social, que para la programación y coordinación de su desarrollo y para la racional prestación de sus servicios públicos requieren una administración coordinada" (Congreso de la República, 1994). En el caso de Bogotá, aunque no se ha reglamentado como un área metropolitana, se considera metrópoli expandida que se encuentra en proceso de integración con los municipios vecinos y que ha venido aumentando la ocupación de territorios aledańos a la capital y zonas de la sabana. En términos del transporte público, la integración regional supone el reto de administrar económica y políticamente los medios de desplazamiento público, tanto existentes como nuevos proyectos, en aras de la productividad social y la competitividad, para las cuales son prioritarios tanto el sistema de este transporte como las obras de infraestructura, y puesto que el territorio se extiende más allá de las áreas metropolitanas tradicionales, requiere de un nuevo marco de gobernabilidad 
regional "capaz de hacer sustentable el desarrollo económico y construir redes de innovación” (Pineda, 2007, p. 89).

La integración intersectorial hace referencia a la coordinación administrativa entre los diferentes gestores de la planificación del sistema de trasporte. Es decir, la relación entre todas las entidades administrativas y gubernamentales que tienen como función la planificación, orientación, control y ejecución de los proyectos sobre la gestión del sistema de trasporte público. Según Pineda, de lo que se trata con la integración multisectorial es de "reconocer la tendencia creciente a la conformación de 'economías en red', en las que se hace evidente la transición de una jerarquía vertical (estructura monocéntrica) a una horizontal (estructura policéntrica, denominada red de ciudades" (Pineda, 2007, p. 91).

La región es el instrumento para el logro del desarrollo sostenible y para materializar las directrices de desarrollo de los organismos internacionales, cuyo escenario político debe construirse sobre la intersección de las funciones de los diversos gobiernos territoriales. Según Pineda, esta

nueva etapa de políticas territoriales, que contemple el relanzamiento del proceso de descentralización en Colombia, exige la adopción de un contrato social entre la nación y las regiones para la concertación de un marco institucional adecuado y el diseño de instrumentos de actuación conjunta y delegada para la promoción regional y local, que puede y debe fortalecerse a partir del enfoque de la gobernanza territorial” (2007, pp. 93-94).

Los retos de la integración intersectorial son los mismos que la gobernanza territorial, que los distintos niveles de gobierno y participación tratan de afrontar desde diversas estrategias. La integración multimodal se refiere a la necesidad de conectar física y virtualmente los medios de transporte de pasajeros. Con el proceso de metropolización, la integración multimodal exige la integración de los diferentes sistemas y componentes existentes funcionando articulada y dinámicamente. Su organización, evolución, gerencia, marco legal, comercialización, tecnología y su infraestructura son determinantes para identificarla como un eslabón de capital importancia en el desarrollo sustentable. Esta integración debe tener en cuenta la realización de trasbordos, la disminución de desventajas para el pasajero, y el impacto ambiental y social. 


\section{Metodología}

La investigación que sustenta esta reflexión es de tipo descriptivo con un enfoque cualitativo. Partiendo de que, en esta clase de estudios, el investigador debe ser capaz de definir qué se va a medir y cómo lograr precisión en esa medición, y que, así mismo, debe ser capaz de especificar quiénes deben estar incluidos en la medición, la metodología consistió en hacer una selección del material documental (documentos de políticas, planes y proyectos de transporte en Bogotá y la región) para hacer un análisis bibliográfico documental que permitió explicar el problema desde una perspectiva crítica, realizar una categorización de las principales variables y plantear lineamientos aplicables a la política local.

\subsection{Revisión documental}

Teniendo en cuenta que esta es una técnica de revisión y de registro de documentos que fundamenta el propósito de una investigación y permite el desarrollo del marco teórico y/o conceptual, fue pertinente en el tipo de investigación realizada: exploratoria descriptiva, etnográfica, teoría fundamentada, pero que aborda todo paradigma investigativo (cuantitativo y cualitativo o multimétodo), por cuanto hace aportes al marco teórico y/o conceptual. Se busca, por medio de esta técnica investigativa, el conocimiento actualizado del tema que se explora, mediante la indagación en los archivos de bibliotecas y hemerotecas, archivos digitales clasificados, revistas y publicaciones registradas y certificadas, archivos documentales de instituciones o grupos reconocidos en el tema de la investigación, etc.

Esta indagación ofreció el conocimiento necesario para comprender el fenómeno del que se ocupa la investigación, además de permitir establecer comparaciones entre los distintos periodos de desarrollo del objeto de la investigación. La revisión documental permitió hacer una retrospectiva del tema en cuestión y plantear comparaciones o relaciones entre las categorías definidas para plantear explicaciones sobre la problemática. Para esto, la revisión documental comprendió tres fases:1) fase de revisión inicial y selección de los documentos, 2) fase de validación y revisión de las referencias seleccionadas; pero, antes de comenzar con el análisis propiamente dicho del contenido, se analizaron las referencias y documentación recopilada con la finalidad de validar la selección previa, y 3) fase de registro y sistematización de la información. 


\subsection{Entrevistas}

Las entrevistas, que fueron de carácter semiestructurado y cualitativa, permitieron describir e interpretar las creencias, las actitudes, los valores y las motivaciones que subyacen a las percepciones de los actores implicados en las políticas de movilidad y transporte en Bogotá, toda vez que "la entrevista cualitativa es entonces el punto de entrada del científico social para comprender el mundo de vida de los informantes, en tanto provee los datos básicos para entender las relaciones entre los actores sociales y sus situaciones" (Bonilla \& Rodríguez, 2005).

La guía para elaborar el cuestionario de las entrevistas se sustentó en la frase mapa, herramienta tomada del enfoque de la teoría de facetas, que propone partir de la discriminación de los roles de los participantes, del ámbito al que pertenecen, de la actitud que se pretende visibilizar en la entrevista, de su participación en el fenómeno investigado (definida como instrumentos) y de las dimensiones de integralidad de la investigación, (gráfica 1).

Gráfica 1. Guía del diseño del cuestionario

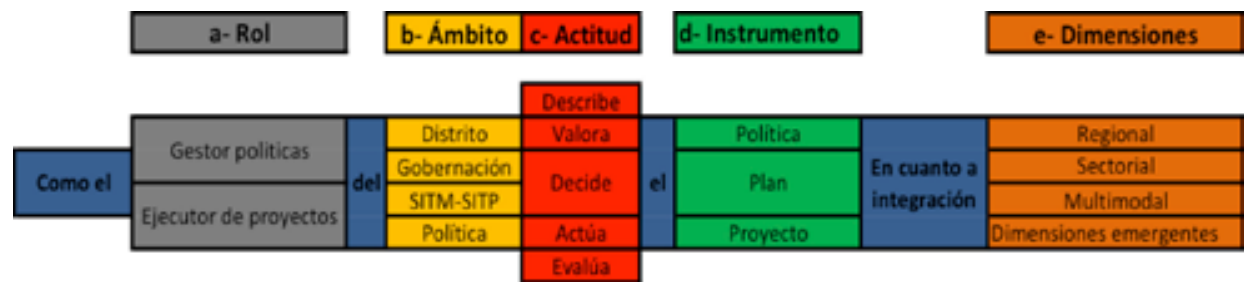

Fuente: elaboración propia

\subsection{Entrevistados}

Con base en esta guía (gráfica 1) se determinó la aplicación del cuestionario a los actores relacionados con la gestión y planificación del transporte en Bogotá ciudadregión, a través de sus voceros autorizados: Transmilenio S. A.-SITP, Secretaría de Región Capital e Integración Regional, Ministerio de Transporte de Colombia, Secretaría Distrital de Movilidad, Secretaría de Movilidad y Transporte del Departamento y Secretaría Distrital de Planeación. 
Juan Manuel González Trujillo Una reflexión sobre el paradigma de la integralidad en el transporte público de Bogotá

\subsection{Determinación de las preguntas}

Divididas en preguntas primarias y en preguntas exploratorias y de síntesis se determinaron, en las primeras:

\subsubsection{Interrogantes sobre integración intersectorial}

Pregunta 1: ¿Cuál es la relación o las funciones de la entidad que usted representa con el tema de transporte masivo de pasajeros y movilidad en la ciudad?

\subsubsection{Interrogantes sobre desarrollo de politicas e iniciativas intersectoriales de movilidad e integración del transporte en Bogotá}

Pregunta 2: ¿Cuáles son las políticas o iniciativas en materia de transporte masivo que se vienen ejecutando actualmente en el estamento al que usted pertenece?

\subsubsection{Interrogantes sobre la materialización de la visión de la integralidad del transporte público en el Sistema Integrado de Transporte Público}

Pregunta 3: ¿Qué es el Sistema Integrado de Transporte Público (SITP), cuáles son los organismos reguladores y qué papel juega en su implementación la entidad que usted representa? Pregunta 4: ¿Qué problemas considera que enfrenta la relación o coordinación de su entidad con las otras entidades o instituciones que tienen que ver con la implementación del SITP?

\subsubsection{Interrogante sobre la integralidad del transporte público T3}

Pregunta 5: ¿Qué entiende por integralidad del transporte masivo de pasajeros y cómo ve la aplicación de este concepto al modelo de transporte que se viene implementando en la movilidad de la ciudad de Bogotá?

\subsubsection{Interrogantes sobre la visión intersectorial en temas clave del SITP T3}

Pregunta 6: ¿Qué conocimiento tiene sobre la captación y el manejo de los recursos para la implementación del SITP y cómo ve la relación entre los gestores del proyecto y los niveles de gobierno? Pregunta 7: ¿Qué conocimiento tiene sobre la posibi- 
lidad de utilizar vehículos eléctricos o híbridos en el SITP y/o sobre los sistemas de cables aéreos como parte de las propuestas de servicios? Pregunta 8: ¿Cómo entiende el manejo de la estructura tarifaria y del sistema de recaudo en la propuesta del SITP y qué posición tiene al respecto?

En las exploratorias y de síntesis se determinaron la Pregunta 9, En su opinión, ¿qué aspectos de educación, cultura ciudadana o responsabilidad social debe contemplar la implementación del SITP para que su desarrollo y aceptación por parte de los usuarios sean adecuados? y la Pregunta 10, ¿Qué reflexiones o preocupaciones le suscita el tema de la implementación del SITP o, en general, la problemática de la movilidad en la ciudad de Bogotá?

\subsection{Procedimiento para el análisis de la información}

Los datos y las informaciones obtenidos, mediante las técnicas del análisis documental y de las entrevistas se codificaron el programa Atlas Ti, (programa informático para el análisis cualitativo de datos). En el análisis documental se partió de dimensiones iniciales de integración, aunque se abrió la posibilidad de una codificación abierta para categorías emergentes.

Después de la codificación, se elaboró un análisis de cada dimensión, de las relaciones y de las jerarquías entre las dimensiones, con los mapas de relaciones que arrojó el software de análisis, para sustentar el estudio de los resultados.

En el análisis de las entrevistas, con base en el recurso aplicado, se fijó la atención sobre el significado del concepto de integralidad, frente al desarrollo del modelo transporte que se viene implementando en la ciudad de Bogotá, partiendo de los procesos de innovación en la política pública, en general, de su incidencia en la política social en particular, y del análisis de los mecanismos de implementación de estas políticas.

La información obtenida con base en la metodología aplicada proporcionó un conocimiento pertinente acerca de la realidad del fenómeno en materia del transporte integral que se está gestando para Bogotá, desde la perspectiva de integralidad en planificación de los sistemas de transporte, en términos de la revisión de las políticas de 
orden internacional, nacional, distrital y local, específicamente de los instrumentos de planificación, para realizar la descripción y el estudio crítico de la situación del ordenamiento territorial y de la aplicación de sus normativas legales e instrumentos técnicos de planificación y gestión territorial.

\section{Resultados}

La presentación de estos resultados se divide en las dimensiones señaladas: integración regional, integración intersectorial e integración multimodal, y presenta, por una parte, el análisis de las fuentes documentales, y por otra, el de las fuentes entrevistadas, para sustentar el balance interpretativo de este trabajo. Debe aclararse que en la revisión de la integración regional no se incluye el análisis de las fuentes, porque las iniciativas de esta dimensión son por ahora incipientes en términos de proyectos o planes relacionados con el transporte público de pasajeros, por lo que no fue posible formular preguntas para las fuentes consultadas cuyas respuestas reflejaran una posición institucional.

\subsection{Revisión documental: lineamientos generales del marco regulatorio de la movilidad y el transporte público para Bogotá}

Puede considerarse como la directriz más general de la movilidad y el transporte, la Ley 769 de 2002 que rige el Código Nacional de Tránsito actual, cuyos principios giran en torno a la calidad, el cubrimiento y la libertad de acceso a la movilidad. Pese a la naturaleza nacional de esta ley, deja diversas facultades en manos de las autoridades de tránsito locales y de los entes territoriales, para regular el tránsito en los aspectos vehicular y peatonal. Esta autonomía se sanciona en el artículo 209 de la Constitución Política, en la Ley 489 de 1998, en la Ley 105 de 1993, en la Ley 336 de 1996, y en el artículo 38 del Decreto Nacional 1421 de 1993.

El Plan Maestro de Movilidad para Bogotá (PMM) empezó a gestarse desde 1993, cuando la administración distrital contrató la asesoría del gobierno de Japón, a través de la Japan International Cooperation Agency (JICA). Esta propuesta inicial sustentó las bases para definir el Sistema Integrado de Transporte (SIT), el Plan de 
Transporte (PIT) y el Sistema Integrado de Transporte Masivo (SITM), cuya configuración incluía el servicio de buses para la ciudad y la región, un sistema de trenes suburbanos o de cercanías, el proyecto Metrobus (actualmente Transmilenio), el proyecto de troncales, el mejoramiento de la malla vial y la posibilidad de construir el metro; se contemplaron 67 proyectos de inversión a corto, mediano y largo plazo, acogiendo la estructura financiera hecha por el JICA.

El Consejo Nacional de Política Económica y Social (Conpes), dentro del Programa Nacional de Transporte Urbano (PNTU) que se generó en respuesta a los retos y desafíos de la movilidad urbana en las ciudades, ha venido liderando estrategias de política pública tendientes a contribuir al mejoramiento de los sistemas de transporte público urbano en las principales ciudades del país, consignadas en los documentos: Conpes 3167, "Política para Mejorar el Servicio de Transporte Público Urbano de Pasajeros" del 27 de mayo de 2002; Conpes 3260, "Política Nacional de Transporte Urbano y Masivo" del 15 de diciembre de 2003 y, con respecto a la ciudad-región Bogotá, los Conpes 3256, "Políticas y Estrategias para la Gestión Concertada del Desarrollo de la Región Bogota-Cundinamarca", del 15 de diciembre de 2003, y Conpes 3677, "Política de Movilidad Integral para la Región Capital Bogotá-Cundinamarca" dl 19 de julio de 2010.

En los Conpes 3167 y 3260, el Consejo define políticas y estrategias para incentivar a las entidades locales a que adopten iniciativas para mejorar los sistemas de transporte público de pasajeros. El Conpes 3256 se centró en el diseño de un marco normativo e institucional adecuado para la construcción de un modelo para unificar los intereses y ventajas competitivas de la ciudad-región. La relación con la gestión urbana se materializa en el marco de ley, por la Ley 388 de 1997 que, para garantizar un desarrollo urbano integral y planificado, exige políticas y planes por parte de los municipios y distritos que sustenten la prestación de servicios, la provisión de infraestructuras y la coordinación interinstitucional.

Estos lineamientos nacionales, en relación con el transporte público en Bogotá, se materializan en el Plan de Ordenamiento Territorial (en su primera versión del Decreto Distrital 619 de 2000, su revisión mediante el Decreto 469 de 2003 y la compilación contenida en el Decreto 190 de 2004) que establece que el sistema de movilidad debe responder a los requerimientos de movilidad de pasajeros y de carga 
Juan Manuel González Trujillo Una reflexión sobre el paradigma de la integralidad en el transporte público de Bogotá

en la zona urbana y de expansión, en el área rural, y la conexión en la ciudad región, con el resto del país y con el exterior.

La aplicación del sistema de movilidad definido por el POT y la implementación del PMM deben lograrse, según la Ley 388 de 1997, a través de la coordinación entre el Departamento Administrativo de Planeación Distrital, la Secretaria de Movilidad (en reemplazo de la anterior Secretaria de Tránsito y Transporte de Bogotá) y las demás entidades que tengan injerencia en el sistema. Mediante el Decreto 319 de 2006, el Distrito adoptó el Plan Maestro de Movilidad (PMM) como el instrumento de planificación de la movilidad que se articula con la estrategia de ordenamiento de la ciudad-región. El PMM busca impulsar una política sectorial para Bogotá, basada en una movilidad segura, equitativa, sostenible y competitiva, estableciendo como eje estructurador el sistema de transporte público. Posteriormente, el Decreto 486 de 2006 le asignó a Transmilenio S.A. las funciones de integrar, evaluar y hacer el seguimiento de la operación del Sistema Integrado de Transporte Público (SITP).

\subsection{Resultados del análisis de las dimensiones de la integralidad a partir de la entrevista con los actores implicados}

El análisis de las respuestas obtenidas en las entrevistas realizadas a los actores involucrados en la gestión y planificación del transporte público masivo en Bogotá, de acuerdo con las dimensiones de la integralidad, permite determinar los siguientes resultados.

\subsubsection{Visión de la coordinación intersectorial en la movilidad e integralidad para las entidades consultadas}

El análisis de este factor se realizó con base en la información suministrada por las fuentes consultadas en respuesta a la pregunta $1:$ ¡Cuál es la relación o las funciones de la entidad que usted representa con el tema de transporte masivo de pasajeros y movilidad en la ciudad?

La fuente de la Secretaria Distrital de Movilidad señaló el Decreto 319 de 2006 que expidió el Plan Maestro de Movilidad para Bogotá como "la carta de navegación 
de sistema distrital de movilidad". La fuente de la Secretaria Distrital de Planeación, en respuesta a la misma pregunta, también se refirió al Acuerdo 257 de 2006 para comentar la participación de la entidad, e hizo una referencia general al subsistema de transporte en relación con la estructura de la ciudad. La fuente de la Secretaría Departamental de Movilidad y Transporte se refirió al Decreto Ordenanzal 00260 de 2008 para señalar que la entidad se define por ser:

la dependencia rectora de las políticas del sector de tránsito y transporte, seguridad e infraestructura vial y de transporte en el Departamento en sus distintos modos carretero, marítimo, fluvial, férreo y aéreo, y tiene como misión orientar y liderar la formulación de las políticas del sistema de movilidad y asegurar la organización y mantenimiento del orden en materia de tránsito y transporte, garantizando la preservación y el mejoramiento de la seguridad y la calidad de vida de la comunidad en el Departamento.

Las demás fuentes no hicieron claridad sobre los marcos regulatorios y se refirieron unas a la integración entre el transporte distrital y regional, y otras, a la integración de los modos de transporte público, masivo y colectivo, en la ciudad (fuente de Transmilenio-SITP) como finalidades de las entidades que representan en relación con el tema señalado.

\subsubsection{Visión del desarrollo de políticas e iniciativas intersectoriales de movilidade integralidad del transporte en Bogotá para las entidades consultadas}

Este análisis se realizó con base en las respuestas de las fuentes consultadas a la pregunta 2: ¿Cuáles son las políticas o iniciativas en materia de transporte masivo que se vienen ejecutando actualmente en el estamento al que usted pertenece?

La fuente de la Secretaria Distrital de Movilidad se refirió al artículo 12 del Plan Maestro de Movilidad y a la Ley 310 de 1996 para señalar que el trabajo que la entidad ha venido desarrollando en este tema ha consistido básicamente en implementar estudios pertinentes con la factibilidad del transporte público integrado.

La fuente de la Secretaria Distrital de Planeación se refirió al Plan de Ordenamiento Territorial (POT) y al Decreto 398 de 2009 para reseñar la iniciativa de la red de 
metros, resultante de los estudios técnicos arrojados por la consultoría de Sener (empresa de ingeniería, construcción e integración de sistemas española) para orientar las estrategias de planificación, gestión y financiamiento de los sistemas de transporte masivo.

La fuente de la Secretaría de Movilidad y Transporte Departamental se remitió al documento Conpes 3677 para señalar las iniciativas que viene desarrollando: evaluación de los avances del sistema integrado de transporte público (SITP), de Transmilenio, de la primera línea del metro y del tren de cercanías. La fuente de la Secretaría de Región Capital e Integración Regional de la Gobernación de Cundinamarca también se refirió al proyecto del tren de cercanías, puesto que la iniciativa principal de la entidad se ha centrado en su estructuración técnico-financiera.

La fuente de Transmilenio-SITP destacó que la iniciativa principal de la entidad ha sido la implementación del sistema integrado de transporte público, que partiendo del modelo de transporte de Transmilenio, busca integrar en un solo esquema el modelo de transporte, bajo el mecanismo contractual de la concesión.

En tanto que la fuente del Ministerio de Transporte señaló los documentos Conpes derivados del Programa Nacional de Transporte Urbano, dentro de un contexto que propone soluciones integrales de movilidad que contemplan el mejoramiento de la calidad de vida, la sostenibilidad ambiental, la coordinación en la ordenación del territorio, el desarrollo urbano, la promoción de tecnologías limpias, entre otros factores, perspectiva en la que señaló la importancia de coordinación institucional entre entidades del ámbito central, regional y local.

\subsubsection{Visión de la integralidad del transporte público para las entidades consultadas}

Respecto de este tema, enfocado en la pregunta 5: ¿qué entiende por integralidad del transporte masivo de pasajeros y cómo ve la aplicación de este concepto al modelo de transporte que se viene implementando en la movilidad de la ciudad de Bogotá?, la fuente de la Secretaria Distrital de Movilidad, se refirió al cambio hacia una operación del transporte público organizada, regulada y controlada que significa la implementación del SITP, y destacó que en ese sentido la integralidad es la conquista de aspectos como la confiabilidad, la regularidad y el nivel de servicio al usuario. 
La fuente de la Secretaria Distrital de Planeación, ofreció una definición de sistema integrado en términos de una "estructura de gestión de transporte que posibilite la movilidad sobre los principios de intermodalidad, lo que significa utilizar los modos de transporte masivo y colectivo bajo un esquema de tarifa integrado, en el que el costo para el usuario tiende a ser eficiente".

La fuente de la Secretaria de Movilidad y Transporte Departamental hizo énfasis en el desarrollo sostenible para definir la integralidad como un deber ser de los planes maestros de movilidad regional en términos de proyectos de infraestructura, de gestión, de manejo, de demanda y oferta de transporte, ligado a las estrategias de la Agenda Regional de Movilidad.

La fuente de la Secretaría de Región Capital e Integración Regional de la Gobernación de Cundinamarca se centró en el rol del usuario para definir la integralidad como "la posibilidad de contar con opciones de transporte público combinadas y coordinadas, sin que medie la diferencia de precios o la competencia entre los sistemas que constituyen la oferta actual". La fuente de Transmilenio-SITP se refirió a la integralidad como "la coordinación de los aspectos jurídicos, financieros, tarifarios y tecnológicos".

\subsubsection{Visión de las entidades consultadas sobre el Sistema Integrado de Transporte Público (SITP)}

Este análisis se realizó con base en las respuestas de las fuentes consultadas a la pregunta 3, ¿Qué es el sistema integrado de transporte público (SITP), cuáles son los organismos reguladores y qué papel juega en su implementación la entidad que usted representa?, y a la pregunta 4, ¿Qué problemas considera que enfrenta la relación o coordinación de su entidad con las otras entidades o instituciones que tienen que ver con la implementación del SITP?

Respecto de la pregunta 3, la respuesta de la fuente de la Secretaria Distrital de Movilidad se centró en la anterior definición y en el señalamiento de las entidades reguladoras: esta Secretaría:

actuará como la autoridad del sistema integrado de transporte público en Bogotá D.C. y sus funciones estarán dirigidas especialmente a la formulación de 
la política pública sectorial, regulación, vigilancia y control de las actividades de tránsito y transporte, coordinación de las instancias de ejecución de dicha política pública sectorial, funciones que ejercerá con el acompañamiento permanente del Comité Sectorial de Desarrollo Administrativo de Movilidad.

La fuente de la Secretaría Distrital de Planeación coincidió en los mismos aspectos de definición y responsabilidad reguladora y señaló que su conformación se apoya en los principios constitucionales de coordinación y complementariedad y ha sido producto de un proceso de integración institucional que se traduce en la integración operacional y tarifaria.

La fuente de la Secretaría de Movilidad y Transporte Departamental se remitió a la definición del sistema, sin agregar otras consideraciones, y la fuente de la Secretaría de Región Capital, sin entrar en la definición, destacó que su alcance se vincula con la región. La fuente de Transmilenio-SITP, sorpresivamente, no dio respuesta a la pregunta. Por su parte la fuente del Ministerio de Transporte señaló el modelo en el que se apoya el proyecto Transmilenio y destacó que aunque el SITP: "no involucra grandes inversiones con corredores troncales, sí involucra un concepto de modernización de todo el sistema con formalización empresarial, con modernización en la flota, con sistemas de tecnología, con recaudos centralizados; con una tarifa baja vas a poder circular por todos los medios de transporte incluido metro, incluido los otros modos".

Con respecto a la pregunta 4, ¿Qué problemas considera que enfrenta la relación o coordinación de su entidad con las otras entidades o instituciones que tienen que ver con la implementación del SITP?, la fuente de la Secretaría Distrital de Movilidad no identificó problemas; la fuente de la Secretaría Distrital de Planeación señaló que los problemas se encuentran en el aspecto de la coordinación institucional, dado el divorcio entre la planeación urbana y la planeación del transporte "que mantienen las tensiones centro-periferia que el sistema de movilidad tiende a privilegiar".

La fuente de la Secretaría de Movilidad y Transporte Departamental señaló como principal problema el escaso conocimiento de algunas de las entidades que lideran la implementación del sistema desde el interior del Distrito; la fuente de la Secretaría de Región Capital e Integración Regional de la Gobernación de Cundinamarca coinci- 
dió en el asunto de la coordinación y destacó la necesidad de que entre en funcionamiento la autoridad regional que estipula el documento Conpes 3677 para afrontar dicha problemática. La fuente de Transmilenio-SITP, sin referirse específicamente a la existencia de problemas, señaló los retos de la intermodalidad en términos de las funciones de las entidades vinculadas, particularmente Transmilenio y la Alcaldía Mayor en lo que respecta a la naturaleza contractual de los prestadores del servicio de transporte público; igualmente, la fuente del Ministerio de Transporte, sin señalarlo como problema, destacó el asunto de la descentralización en el tema de tránsito y transporte como retos en las decisiones estratégicas, en la inversión, en la expansión con respecto a los entes gestores y cofinanciantes.

\subsubsection{Visión de la integración multimodal de las entidades consultadas sobre temas clave del SITP}

En la integración multimodal se destacan por su importancia los temas del financiamiento del SITP, la implementación de nuevas tecnologías en los vehículos y la integración tarifaria, que son recurrentes en los interrogantes que surgen en la sociedad en general, por lo que fueron enfocados a partir de las preguntas 6, ¿Qué conocimiento tiene sobre la captación y el manejo de los recursos para la implementación del SITP y cómo ve la relación entre los gestores del proyecto y los niveles de gobierno?, 7, ¿Qué conocimiento tiene sobre la posibilidad de utilizar vehículos eléctricos o híbridos en el SITP y/o sobre los sistemas de cables aéreos como parte de las propuestas de servicios? y 8 , ¿Cómo entiende el manejo de la estructura tarifaria y del sistema de recaudo en la propuesta del SITP y qué posición tiene al respecto?, bajo el supuesto de que la claridad que sobre estos tienen las fuentes consultadas, debe reflejarse por principio en las diversas manifestaciones que hacen sobre el tema $y$, por ende, en el conocimiento que ayudan a crear y fortalecer en la ciudadanía.

En la respuesta a la pregunta 6 , las fuentes consultadas coincidieron en la falta de conocimiento o claridad sobre el factor interrogado; incluso la fuente de la Secretaría de Movilidad y Transporte Departamental manifestó no haber recibido ninguna orientación departamental al respecto $y$, sin ofrecer referencias concretas la fuente Transmilenio-SITP se refirió a las ventajas de un sistema de transporte que le permita al usuario "hacer distintos viajes sin tener que cambiar de sistema". 
Con respecto a la pregunta 7 , solo dos de las fuentes aportaron reflexiones pertinentes sobre el factor: la fuente de Transmilenio-SITP que destacó el contenido de la concesión de Transmilenio en términos de su capacidad para implementar y hacer seguimiento y control de todo tipo de tecnología en los distintos niveles de la infraestructura de transporte, destacando el de la información del servicio al usuario, y la fuente del Ministerio de Transporte que señaló que el Gobierno nacional cuenta con los recursos suficiente para fomentar y apoyar el uso de tecnologías limpias.

En la pregunta 8, integración tarifaria, solo la fuente de la Secretaría Distrital de Movilidad demostró tener claridad al respecto, al remitirse a los análisis realizados por los especialistas en la materia, quienes estructuraron los modelos financieros para la operación del SITP a través de la Concesión de Zonas Operacionales de Transporte, con delegación de flota y rutas, señalando que el sistema de recaudo centralizado que se ha adoptado garantiza la evasión y una distribución de los recursos de acuerdo con el modelo financiero de base.

\subsection{Balance interpretativo sobre el reconocimiento del paradigma de integralidad en la gestión y planificación del transporte público en Bogotá, ciudad-región}

A continuación se hace una reelaboración de las dimensiones de la integralidad y de sus relaciones entre sí, para sustentar que, respecto de los retos de gestión que implica la planificación de la movilidad y el transporte, no existe consenso sobre el significado de este paradigma, como uno de los conceptos más importantes dentro del desarrollo, y en particular de Bogotá, ciudad-región.

El análisis documental y de las entrevistas permite afirmar que los distintos entes administrativos, distritales y regionales han tratado de materializar las estrategias propuestas en el Decreto 295 de junio de 1995: Plan de Desarrollo Económico Social y de Obras Públicas, "Formar ciudad", que con base en los estudios de la Japanese International Cooperation Agency (JICA), sentó el marco conceptual para dar respuesta a las necesidades de movilidad de los habitantes de la ciudad-región en el área urbana e interurbana, porque en este estudio se determinaron mecanismos que 
han venido siendo implantados como el Sistema Integrado de Transporte, el Plan Maestro de Movilidad y el Sistema Integrado de Transporte Público (representado en la actualidad por Transmilenio), en un marco de integración de las políticas de transporte, tránsito y obras viales.

Puede, entonces, afirmarse que la integralidad es el lineamiento principal de este derrotero y que está presente en los marcos regulatorios y en las iniciativas que lo han intentado materializar a través del tiempo; pero, con base en el análisis es posible concluir que en la política de integralidad hay al menos cinco formas de concebir la integración: 1) como integración de políticas, en términos del uso del suelo e impacto ambiental; 2) como integración institucional que se refiere a la cooperación interinstitucional y de los actores del sector; 3) como integración operacional que se refiere a la articulación de los diferentes servicios; 4) como integración estratégica entre los modos de transporte y su infraestructura física, de gestión y tarifaria, y 5) como integración estructural, en términos del espacio y los servicios público, la vivienda y el equipamiento de la ciudad.

La integración intersectorial parece concebirse en un principio, y exclusivamente, como la integración entre la planificación del transporte y la planificación territorial, pero el análisis de la información recogida revela otra acepción de la integralidad dentro de esta dimensión: la integralidad interinstitucional, entendida como la necesidad de integración entre instituciones del mismo sector. A este respecto se pueden resaltar, por ejemplo, problemas de coordinación o traslape de competencias entre entidades dentro del sector movilidad como la Secretaría de Movilidad y Transmilenio S.A., y a escala metropolitana aún más con las secretarías de tránsito de los municipios vecinos.

Consolidar un sistema multimodal de transporte a escala metropolitana que equilibre el uso de todos los modos de movilidad es una de las metas y en ello convergen los distintos actores entrevistados en esta investigación, para lo cual, el sistema integral de transporte no se debería limitar al componente masivo y contemplar los conjuntos de medios (motorizados y no motorizados) y modos (individuales y colectivos) de desplazamiento a una escala metropolitana, al menos en cuanto a las necesidades de la ciudad de articular los distintos modos de transporte. 
Las anteriores reflexiones sustentan el surgimiento en este estudio de cuatro dimensiones emergentes del concepto de integración que permiten repensar una adecuada adopción del paradigma de integralidad en relación con las propiedades del sistema de transporte público: integrado e integridad, y con las multidimensionalidades de la movilidad: integración estructural entre los niveles de gobernabilidad y la articulación entre el sector público y el privado.

\section{La integración estructural}

Entendida como la propiedad que tiene un sistema de transporte de hacer parte de un todo, obedece a una visión sistémica de la ciudad, conformada por varios subsistemas que coexisten armónicamente entre sí, incluida la articulación con la infraestructura urbana que no pertenece al sistema de transporte: servicios públicos, equipamientos, vivienda.

\section{Integración con las multidimensionalidades}

En las entrevistas se revela que la mayor necesidad en los sistemas de transporte es precisamente el intercambio multimodal, esto es, la posibilidad de aprovechar los distintos modos de transporte en la movilidad de las personas, bajo un esquema integrado que tenga en cuenta factores sociales, medioambientales y económicos en términos tanto generales como del usuario.

\section{Integración como integridad}

En las entrevistas se visibiliza una acepción que excede las dimensiones de partida, y es que hay actores que conciben la integralidad dentro de una acepción que se acerca más al concepto de íntegro que al de integral o integrado, relacionada con un sistema de movilidad competente, funcional y con cobertura completa: un sistema "íntegro". En cuanto a la integración referida a un sistema que se integra con otros sistemas, se refiere a la posibilidad del transporte público de integrar a todos los miembros de la sociedad y darles acceso a la movilidad. 


\section{Integración entre el sector público y el privado}

Referida a un sistema que se integra con otros sistemas, se refiere a la posibilidad del transporte público de integrar a todos los miembros de la sociedad y darles acceso a la movilidad.

\section{ConClusiones}

En este artículo, y con base en el trabajo investigativo en el que se sustenta, se evidencia que el paradigma de integralidad emanado por los organismos internacionales, y específicamente por la Cepal, como una estrategia de desarrollo que permite sustentar la planificación del transporte y la movilidad en América Latina y el Caribe, no ha tenido una adecuada comprensión y aplicación en los planes y programas adoptados para este sector en Bogotá, siendo uno de sus principales causas el debilitamientos del rol de Estado, desde la descentralización política instaurada por la Constitución Política de 1991.

Esta condición se refleja en dos fenómenos que afectan la eficacia de las políticas e iniciativas en el sector de la movilidad y el transporte público y por ende, también la provisión eficiente tanto de la infraestructura como de los servicios: por un lado, la ausencia de acciones coordinadas entre los planificadores y gestores de los distintos componentes del sistema de transporte público: infraestructura, logística y regulación y control, lo que conlleva a la dispersión y multiplicidad de posiciones porque los derroteros se adoptan desde distintas visiones sectoriales, y por otro, la falta de criterios comunes respecto del diseño y ejecución de tales políticas y planes que hace que la mayoría de iniciativas carezcan de sostenibilidad y continuidad, porque la segmentación de las actividades y de las responsabilidades afecta la interrelación de los componentes del sistema de transporte público que en realidad están interrelacionados por su propia naturaleza sistémica, provocando actuaciones descoordinadas entre los sectores involucrados, tanto públicos como privados.

Dentro de las estrategias de desarrollo con las que se ha asociado la problemática de la movilidad y el transporte público en este trabajo, integración regional, integración intersectorial e integración multimodal, pareciera que la más relacionada con el sector 
es la integración multimodal, referida a la adecuada articulación entre los distintos modos de transporte, y es a la que le apunta, por ejemplo, la consolidación del Sistema Integrado de Transporte Público (SITP) en la ciudad, pero las soluciones a la movilidad que pueda ofrecer este sistema no pueden ser sino parciales en tanto no se unifiquen las políticas y procesos de gestión en un marco de movilidad regional capaz de articular los esquemas operacionales de los sistemas de transporte, en esta caso público, con el modelo de desarrollo regional que se impone en América Latina y el Caribe.

Incluso, la eficacia del modelo de sistema de transporte masivo para Bogotá que se apoya en Transmilenio como eje estructurante del Sistema Integrado de Transporte Público (SITP) genera dudas en varios expertos, entre ellos Silva (2010), sobre su capacidad de potenciar la desconcentración de la ciudad central y fortalecer centralidades en la periferia para equilibrar el desarrollo de la ciudad a largo plazo, uno de los mandatos del POT, si se sigue manteniendo el esquema de que su organización dependa de la demanda y entre tanto no se logre la articulación con los distintos modos de transporte y el manejo adecuado de la malla vial intermedia.

Para Silva, este modelo de transporte masivo refuerza el patrón de desarrollo urbano marcado por la polarización de la accesibilidad y segregación de los usos del suelo, debido a la sectorización en la planeación del sistema que dificulta la articulación de los diferentes sistemas y modos de transporte (Silva, 2010, p. 61), y se puede concluir de su reflexión que se demanda una instancia política y administrativa capaz de articular e integrar los planes y proyectos que relacionan la movilidad y el transporte con el ordenamiento de la ciudad, función que debiera cumplir la Secretaria de Planeación Distrital, pero que no puede encarar porque su papel ha quedado relegado a generar norma urbana (Silva, 2010).

Lo anterior permite sustentar que el modelo de desarrollo que impone el modelo de ciudad región, parece inclinarse hacia la conformación del área metropolitana para Bogotá, uno de los objetivos del plan de desarrollo de la actual administración distrital, pues esta figura administrativa estaría en capacidad de vincular la acción coordinada de los municipios para lograr el desarrollo equilibrado del territorio y la ejecución de las obras y proyectos que requiere, entre otros sectores, el de la movilidad y la planificación del transporte público. Esta estrategia sería la única capaz de sustentar y aplicar políticas integradas y sostenibles de infraestructura, logística 
y movilidad para afrontar los retos actuales y futuros de la ciudad-región, pero tal estrategia depende del fortalecimiento de la institucionalidad, a través de la coordinación y coherencia entre las instancias intersectoriales, y que estas estén ligadas a una política general que supere los límites de las administraciones que participan en la esfera municipal, esto es, la existencia de marcos regulatorios comunes de infraestructura, logística y regulación multidisciplinarios y coordinados entre los diversos sectores y actores públicos y privados.

La recomendación principal derivada de la investigación es la creación y fortalecimiento de este nivel de gobierno de escala regional, responsable de coordinar la planeación de la movilidad y del transporte, de su infraestructura y de integrar la operación del transporte público, pues, sin este, el modelo de Bogotá, ciudad-región, no tiene posibilidades de acoger e implementar un verdadero paradigma de integralidad.

\section{REFERENCIAS}

Alcaldía Mayor de Bogotá (2009). Decreto 309 de 2009. Por el cual se adopta el Sistema Integrado de Transporte Público para Bogotá y se dictan otras disposiciones. Recuperado de: http://www.bogota.gov.co/portel/libreria/pdf/decreto309.pdf.

Alcaldía Mayor de Bogotá (2006). Decreto 319 de 2006. Por el cual se adopta el Plan Maestro de Movilidad para Bogotá Distrito Capital, que incluye el ordenamiento de estacionamientos, y se dictan otras disposiciones”. Recuperado de: http://www.alcaldiabogota. gov.co/sisjur/normas/Normal.jsp?i=21066.

Alcaldía Mayor de Bogotá (2006). "Formulación del Plan Maestro de Movilidad para Bogotá. Componente Legal, V. 8”. Recuperado de: http://www.movilidadbogota.gov.co/ hiwebx_archivos/ideofolio/03-ComponenteLegalV8_11_39_12.pdf.

Alcaldía Mayor de Bogotá (2004). Decreto 190 de 2004. Por el cual se compilan las disposiciones de los Decretos Distritales 619 de 2000 y 469 de 2003. Recuperado de: http:// www.alcaldiabogota.gov.co/sisjur/normas/Norma1.jsp?i=13935Colombia.

Alcaldía Mayor Santa Fe de Bogotá (2002). Plan de Ordenamiento Territorial, POT. Departamento Administrativo de Planeación Distrital. Recuperado de: http://www.alcaldiabogota.gov.co/sisjur/normas/Norma1.jsp?i=5002. 
Juan Manuel González Trujillo Una reflexión sobre el paradigma de la integralidad en el transporte público de Bogotá

Bonilla E. y Rodríguez P. (2005). Más allá del dilema de los métodos, la investigación en ciencias sociales (3. ${ }^{a}$ ed.). Bogotá: Grupo Editorial Norma.

Campos, Javier; De Rus, Gines \& Nombela, Gustavo (2003). Economía del transporte. Barcelona: Antonio Bosch.

Cipoletta, Georgina (2011). "Propiciando un cambio de paradigma en las políticas de transporte, integralidad, sostenibilidad y comodidad”. En Boletín FAL, edición 3041, N. ${ }^{\circ} 12$.

Colombia, Congreso de la República (1997). Ley 338 de 1997. Por la cual se modifica la Ley 9 de 1989 y la Ley 2 de 1991, y se dictan otras disposiciones. Recuperado de: http:// www.alcaldiabogota.gov.co/sisjur/normas/Norma1.jsp?i=339.

Colombia, Congreso de la República (1994). Ley 128 de 1994. Por la cual se expide la Ley Orgánica de las Áreas Metropolitanas. Recuperado de: http://www.secretariasenado.gov. co/senado/basedoc/ley/1994/ley_0128_1994.html.

Colombia, Consejo Nacional de Política Económica y Social (2010). Documento Conpes 3677 "Movilidad integral para la región capital Bogotá-Cundinamarca-Bogotá", 19 de julio de 2010. Bogotá: editor.

Colombia, Consejo Nacional de Política Económica y Social (2005). Documento Conpes 3368 "Política nacional de transporte urbanos y masivo". 1 de agosto de 2005. Bogotá: Autor.

Colombia, Consejo Nacional de Política Económica y Social (2003). Documento Conpes 3260, "Politica nacional de transporte urbano y masivo". 15 de diciembre de 2003. Bogotá: Autor.

Colombia, Consejo Nacional de Política Económica y Social (2002). Documento Conpes 3167, "Política para mejorar el servicio de transporte público urbano de pasajeros", 27 de mayo de 2002. Bogotá: Autor.

Comisión Económica para América Latina y el Caribe (2008). "Informe de políticas de movilidad urbana”. En: Boletín FAL, edición N. 260 (abril). Recuperado de: http://www. cepal.org/usi/noticias/bolfall/4/33864/FAL-260.web.pdf.

Comisión Económica para América Latina y el Caribe (1997). Urbanización y metropolización. Recuperado de: http://habitat.aq.upm.es/iah/cepal/a003.html. 
Montezuma, Ricardo (2003). “Ciudad y transporte: la movilidad en Colombia”. En: Balbo, Marcelo; Jordán, Ricardo y Simioni, Daniela (comps.). La ciudad inclusiva. Santiago de Chile: Comisión Económica para América Latina y el Caribe.

Pineda Hoyos, Saúl (2007). Gobernanza territorial e integración en Colombia: la experiencia de Bogotá-Cundinamarca. Recuperado de: http://www.urosario.edu.co/urosario_ files/4a/4ae062c3-ef1e-4551-abf5-2784adede894.pdf.

Rodríguez, Eduardo \& Carvajal, Jorge (2015). Estado, industrialización periférica y política urbana. Sociología urbana brasilera. En Las miradas de la globalización desde el Estado, el Derecho y el Territorio. pp. 121-161. Bogotá: Editorial Universidad Libre.

Sánchez, Silvana. (2010). “Treinta años de aportes de la Cepal a la institucionalidad de la infraestructura de transporte de América Latina y el Caribe”. En Boletín FAL, ed. 292, N. ${ }^{\circ}$ 12. Recuperado de: http://www.cepal.org/usi/noticias/bolfall/5/42745/FAL-292-WEB. pdf

Silva, Liliana. (2010) "El impacto del transporte en el ordenamiento de la ciudad: el caso de Transmilenio en Bogotá”. En revista Territorios, N. ' 22, pp. 33-64.

Woolcott, Olenka. (2016). “Arturo Valencia: un jurista de importancia”. En Problemas actuales del derecho privado y el derecho laboral, pp. 27-37. Bogotá: Editorial Ibáñez. Recuperado de: http://porticus.usantotomas.edu.co/bitstream/11634/1451/1/Problemas\%20actuales\%20del\%20derecho\%20privado\%20y\%20derecho\%20laboral.pdf 